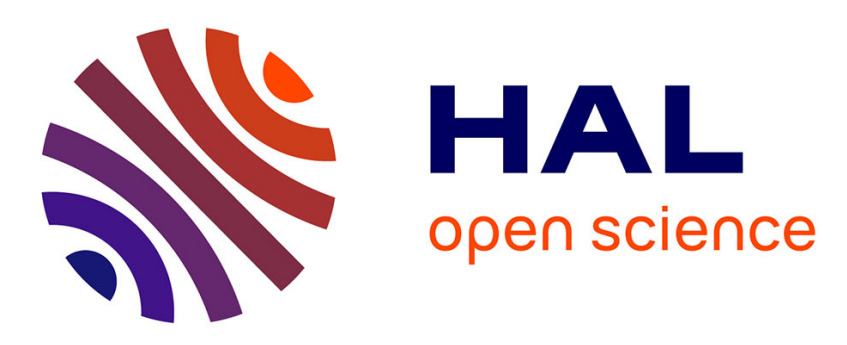

\title{
Dose Measured On-Board INTEGRAL After More Than 12 Years in Space
}

Arnaud Claret, Philippe Laurent, Aymeric Sauvageon, Volodymyr Savchenko

\section{To cite this version:}

Arnaud Claret, Philippe Laurent, Aymeric Sauvageon, Volodymyr Savchenko. Dose Measured OnBoard INTEGRAL After More Than 12 Years in Space. IEEE Transactions on Nuclear Science, 2015, 62, pp.2784 - 2791. 10.1109/TNS.2015.2473671 . cea-01851363

\section{HAL Id: cea-01851363 https://hal-cea.archives-ouvertes.fr/cea-01851363}

Submitted on 30 Jul 2018

HAL is a multi-disciplinary open access archive for the deposit and dissemination of scientific research documents, whether they are published or not. The documents may come from teaching and research institutions in France or abroad, or from public or private research centers.
L'archive ouverte pluridisciplinaire HAL, est destinée au dépôt et à la diffusion de documents scientifiques de niveau recherche, publiés ou non, émanant des établissements d'enseignement et de recherche français ou étrangers, des laboratoires publics ou privés. 


\title{
Dose Measured On-Board INTEGRAL After More Than 12 Years in Space
}

\author{
Arnaud Claret, Member, IEEE, Philippe Laurent, Aymeric Sauvageon, and Volodymyr Savchenko
}

\begin{abstract}
The INTEGRAL satellite was launched on October 17, 2002 and all on-board instruments are still operating successfully after more than 12 years, which represents more than a full solar cycle. This paper deals with the dose measured by 8 RadFETs mounted in 2 electronic boxes, which control the detector plane of the IBIS/ISGRI camera. These measurements are compared to what could be predicted before the launch, by using the dose profile, as determined by common space environment software, and also a detailed sectorial analysis of shielding materials around the RadFETs by means of a Monte-Carlo based model of the whole spacecraft. In this study, the sectorial analysis relies on the accurate knowledge of the geometry, as determined from the qualification model of the electronic box still in the lab. In order to take into account the orbit evolution with time, the dose profile is determined with yearly-averaged orbital parameters for each of the 12 years of measurements. The aim of this analysis is to estimate the possibility of releasing some margins for future projects.
\end{abstract}

Index Terms -Dose, radFET, space environment.

\section{INTRODUCTION}

$\mathbf{T}$ HE INTEGRAL (INTErnational Gamma-Ray Astrophysics Laboratory) satellite is an ESA (European Space Agency) gamma-ray observatory successfully launched from Baikonour on October 17, 2002. It is devoted to the observation of the universe between few $\mathrm{keV}$ up to $10 \mathrm{MeV}$, the energy range of the most extreme phenomena (black holes, accretion in X-ray binaries, gamma-ray bursts...). Two telescopes are mounted on INTEGRAL. The first one, IBIS (Imager on Board the Integral Satellite), provides diagnostic capabilities of fine imaging whereas the second one, SPI (SPectrometer on Integral), performs spectral analysis of gamma-ray sources. Both are coded mask aperture telescopes. In order to achieve a spectral coverage from several tens of $\mathrm{keV}$ to several $\mathrm{MeV}$, the detection unit of the imager IBIS [1] is composed of two gamma cameras, ISGRI (Integral Soft Gamma-Ray Imager) [2] covering the range from $15 \mathrm{keV}$ to $1 \mathrm{MeV}$ and PICsIT (Pixilated CsI Telescope) [3] covering the range from $170 \mathrm{keV}$ to $10 \mathrm{MeV}$. The orbit of INTEGRAL was selected to minimize

Manuscript received July 09, 2015; revised August 20, 2015; accepted August 24, 2015.

A. Claret and A. Sauvageon are with CEA-Saclay, Irfu/SAp, F-91191 Gifsur-Yvette, France (e-mail: arnaud.claret@cea.fr).

P. Laurent is with CEA-Saclay, Irfu/SAp, F-91191 Gif-sur-Yvette, France, and also with Université Paris 7, APC, F-75205 Paris, France.

V. Savchenko is with Université Paris 7, APC, F-75205 Paris, France.

Color versions of one or more of the figures in this paper are available online at http://ieeexplore.ieee.org.

Digital Object Identifier 10.1109/TNS.2015.2473671

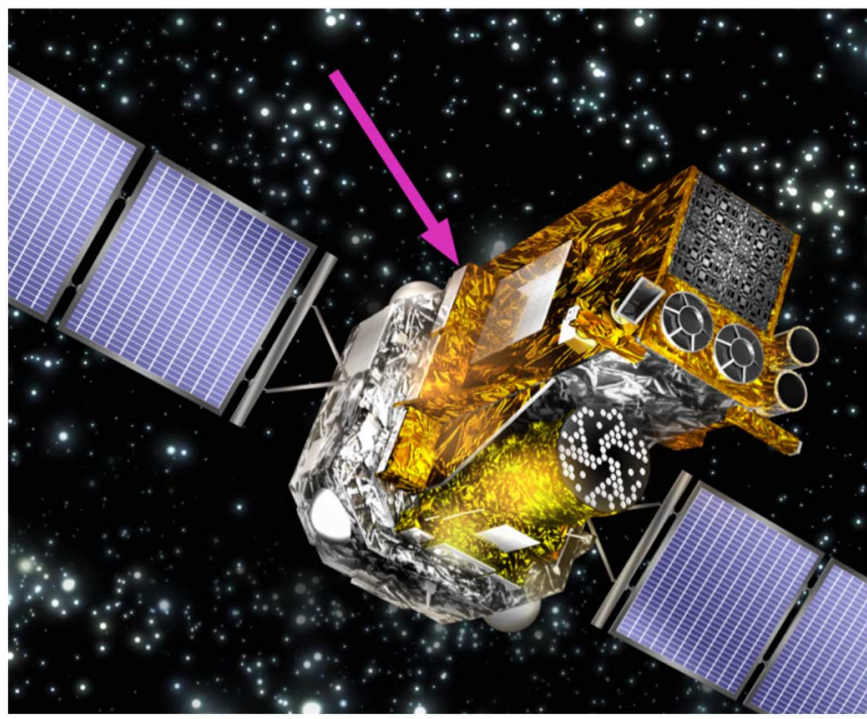

Fig. 1. View of the INTEGRAL satellite showing the two high energy telescopes using the coded-mask imagery technic, SPI at the foreground and IBIS at the background. The pink arrow indicates the location of one of the two MCE boxes, the other one being located at the same level but almost symmetrically with respect to IBIS telescope axis. Adapted from ESA picture.

the background level of instruments, maximizing at the same time its uninterrupted scientific observing time and also the telemetry flow. The satellite was injected on a HEO (72 hours period, perigee $\sim 10,000 \mathrm{~km}$, apogee $\sim 155,000 \mathrm{~km}$ and inclination $\sim 51^{\circ}$ ), where the main contributions to the dose come from trapped particles and solar energetic particles. The scientific observation window is defined by the times of entry and exit of the outer electron belt, the satellite being still directly exposed to cosmic rays and solar particles during $\sim 65$ hours over 72 per orbit.

The present paper deals with data recorded by the MCE (Module Control Electronics) devoted to control the 8 independent modular detection units which compose the large detector plane of the ISGRI camera. An electronic card controls each detector module and the total of 8 cards are grouped in 4 in two MCE boxes that are located beside the IBIS telescope (see Fig. 1). On each card, a RadFET is mounted in order to monitor the deposited dose. The aim of this study is first to show the dose measurements recorded by the IBIS RadFETs and second, to compare them with the predicted value based on information available pre-launch. Before the launch, the dose can be assessed from a sectorial analysis of shielding materials, associated to a dose profile curve issued by space environment software used. The data set is presented in Section II. The 


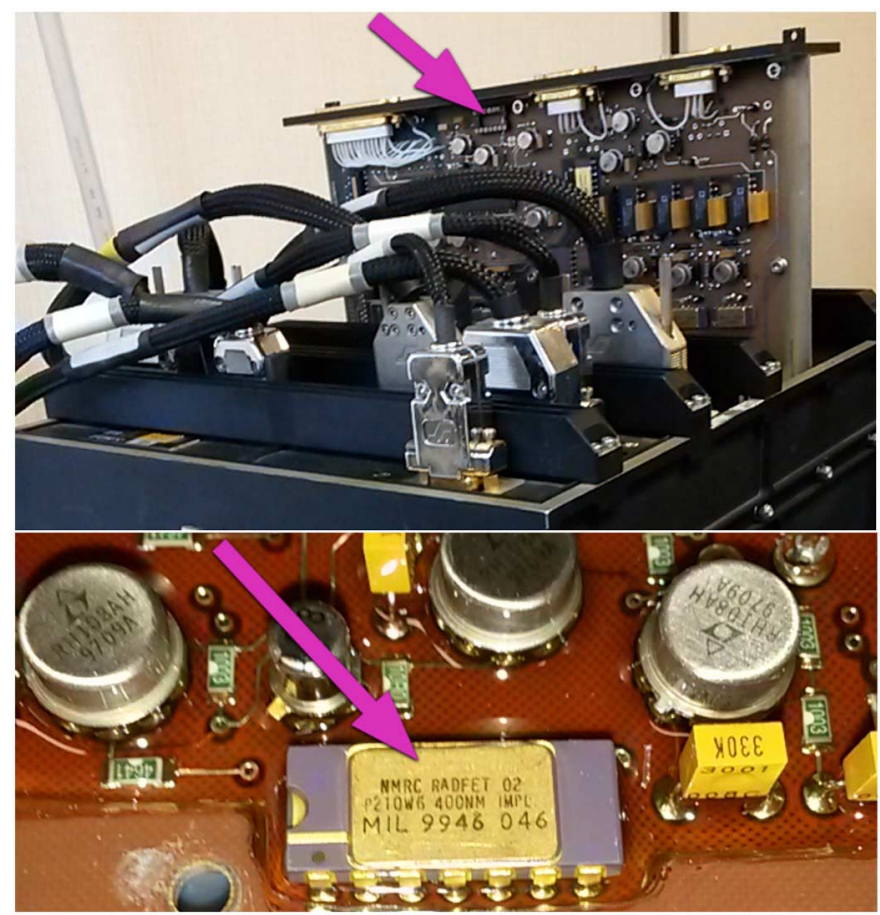

Fig. 2. Photography of the qualification model of the MCE box containing the 4 electronic cards used to control 4 modules of the ISGRI camera. The pink arrows indicate the position of the RadFET component on each card. On the upper panel, the component itself was removed in order to avoid ambient irradiation before the launch. The lower panel shows the RadFET provided by Tyndall and mounted on the spare card.

geometrical model used for determining the shielding material around the RadFETs is described in Section III, followed by the space environmental parameters used to derive the dose profile curve, and the resulting dose map. Section IV deals with the interpretation of the difference between the calculated doses with respect to the one measured in-flight by the RadFETs. Finally, some perspectives of this work are given in the conclusion.

\section{DATA}

A RadFET is a discrete p-channel MOSFET optimized for radiation sensitivity. Through generation and trapping of radiation-induced charges in the gate oxide, radiation exposure changes the output voltage of the RadFET and this change is related to the radiation dose. The RadFET response is non-linear and a pre-recorded calibration curve is used to read the dose. Forcing a DC current into the device and measuring the DC voltage allows the dose read-out. The measurement is sensitive to the temperature of the component itself and varies as the temperature in the cube power. This allows us to consider that the relative error on the measurement of the dose is three times the relative change in temperature of the component $(\Delta \mathrm{D} / \mathrm{D}=$ $3 \times \Delta \mathrm{T} / \mathrm{T}$ ). On-board INTEGRAL, the 8 RadFETs are located at the top center of the electronic cards grouped by 4 in the two MCE boxes, whose upper face is oriented towards the top of the satellite/telescope (see Fig. 2). A probe located at the center of each electronic card monitors its temperature in-flight, which remains at $\sim 20^{\circ} \mathrm{C}$. As visible on Fig. 3, the relative variation of temperature of each card remains lower than $\pm 2.5 \%$ all along the mission. Even if the in-flight temperature of RadFET

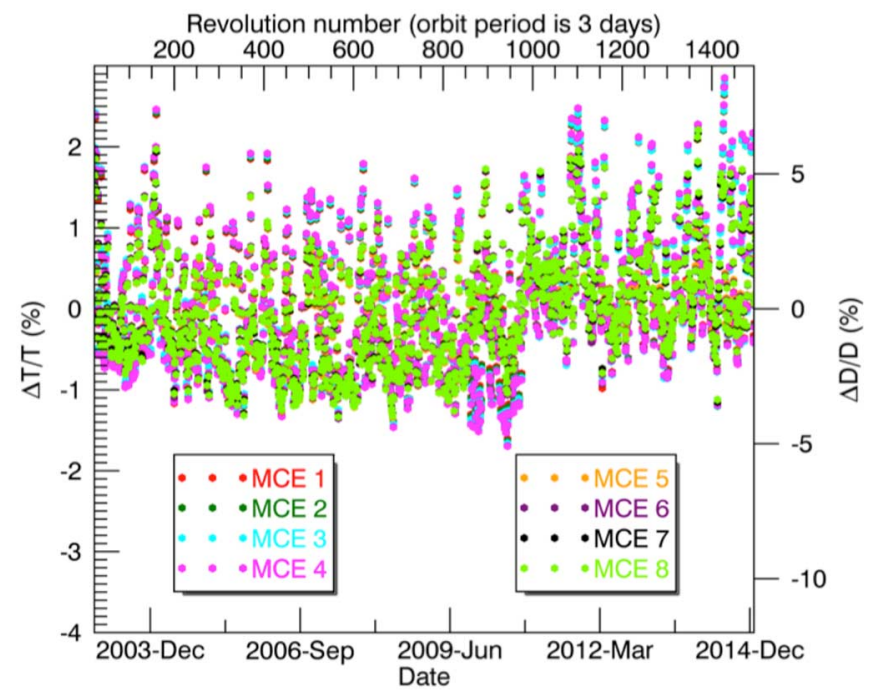

Fig. 3. Evolution of the temperature recorded at the center of the 8 electronic cards where the RadFETs are mounted. The relative variation of the temperature ( $\Delta \mathrm{T} / \mathrm{T}$ on the left axis) is converted into relative error on the measured dose ( $\Delta \mathrm{D} / \mathrm{D}$ on the right axis).

is slightly different from that measured by the probe, the relative variation of temperature $(\Delta \mathrm{T} / \mathrm{T})$ at the location of the RadFET can be considered as the same or similar to that recorded by the probe at the center of the card, and we deduce that the relative error on the dose is $\sim 8 \%$ due to temperature variations. Moreover, the ground calibrations of RadFETs were made at similar temperature. Concerning the self-annealing, which also contributes to modify the response of RadFET [4], we consider that the annealing timescale is several tenth of days at $\sim 20^{\circ} \mathrm{C}$, which is much longer than the time between two passages in the radiation belts. Thus, the RadFETs do not have the time to self-recover, and we do not need to correct for the self-annealing. Finally, we adopt a global relative error of $15 \%$ (divided into $8 \%$ for the temperature variation as determined above + an arbitrary value of $7 \%$ for other possible errors not accounted for).

The values of the dose provided by the ISGRI/RadFETs, sampled once per orbit, are plotted on Fig. 4, together with the dose measured independently on-board INTEGRAL by IREM (Integral Radiation Environment Monitor [5]) and solar cycle. Since the raw values of IREM measurements could not be converted into physical units ( $\mathrm{rad}$ ) due to lack of information required for this conversion, the IREM values were scaled with respect to the mean measurements provided by ISGRI/RadFETs. Nevertheless, this allows checking there is no strong difference in the behavior of these two instruments. On this figure, we can see that at first order, the measured dose after 12 years is of this order of $\sim 1 \mathrm{krad}$.

On Fig. 4, small-scale variations are due to the daily average of values fluctuating with the temperature. The sudden jumps $\geq$ $50 \mathrm{rad}$ are due to solar flares and also to the encoding precision of the voltage measured at the output of RadFETs (two bits for around three hundreds of rad). The goal of the following section is to understand the global evolution of the dose along the time, and compare it to what can be predicted from environmental models. 


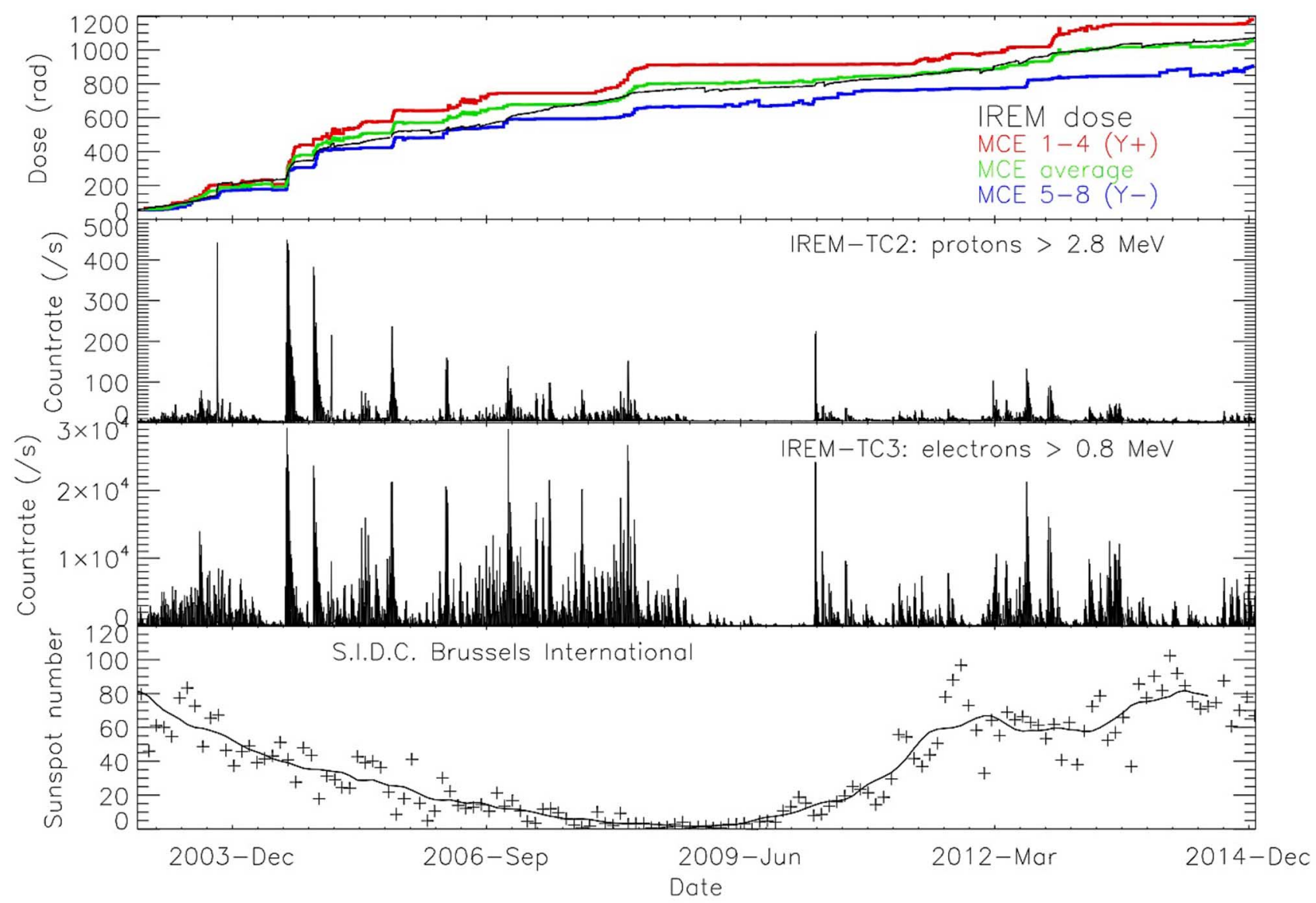

Fig. 4. The top panel shows the integrated dose recorded by ISGRI/RadFETs as a function of time over a 12 years period. The red and blue curves represent respectively the averaged value of 4 RadFETs located in the same electronic box (number 1 to 4 for the first box, number 5 to 8 for the second one). Superimposed in green is the averaged value of all RadFETs, and in black an estimation (see text) of the dose deduced from IREM data. The two middle panels display the ambient particle fluxes recorded independently by IREM also on-board of INTEGRAL, showing a clear correlation with the sudden variations of the dose measured by ISGRI/RadFETs. The lowest panel displays the sunspot number as an indication of the solar cycle.

\section{MODEL}

\section{A. Sectorial Analysis}

Given the complex architecture of the INTEGRAL spacecraft, we used the full Monte-Carlo model (based on Geant3) used to calibrate the response of the instruments, the main advantage being that this very elaborate model has been validated [6] and is widely used to determine the instrumental response and generate the scientific products. Fig. 5 shows where the two MCE boxes are located on the spacecraft at the bottom of IBIS. In the present study, only the geometrical description of the model is used since non-interacting particles (geantinos) were used to determine the thicknesses of all materials from all directions around the ISGRI/RadFETs. No physical interactions are involved in our study. The thickness of any material was reduced to that of Al considering the ratio of densities. Fig. 6 and Fig. 7 show examples of result of our Monte-Carlo simulations, in the form of computed maps of Al equivalent thickness around the RadFETs of MCE 1 and MCE 6, respectively the less and best shielded cards by surrounding materials. In this study, a total of $8 \mathrm{Al}$ thickness maps were computed, one for each RadFET.

Fig. 8 shows the angular referential used for displaying the above map of shielding materials. Note that these axes are not

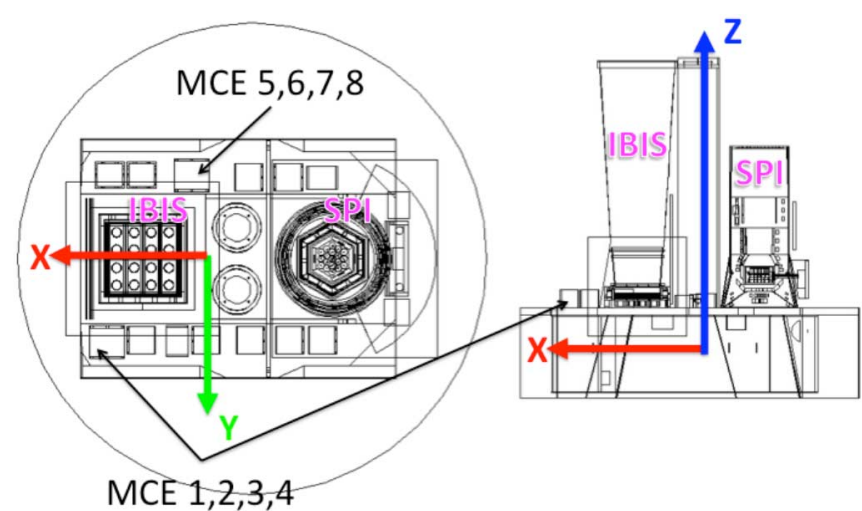

Fig. 5. Top view (left panel) and side view (right panel) of the geometric model of the whole spacecraft as represented in Geant3, where the positions of the two MCE boxes are marked.

the spacecraft mechanical axis, but those used in our MonteCarlo code. The thermal constraints of the instruments require that the satellite maintain the X-axis in the direction of the Sun with a tolerance of $\pm 40^{\circ}$. This is enough to effectively randomize the directional exposure to solar and trapped particles. This allows using omnidirectional average fluxes of particles, as detailed in the following section. 


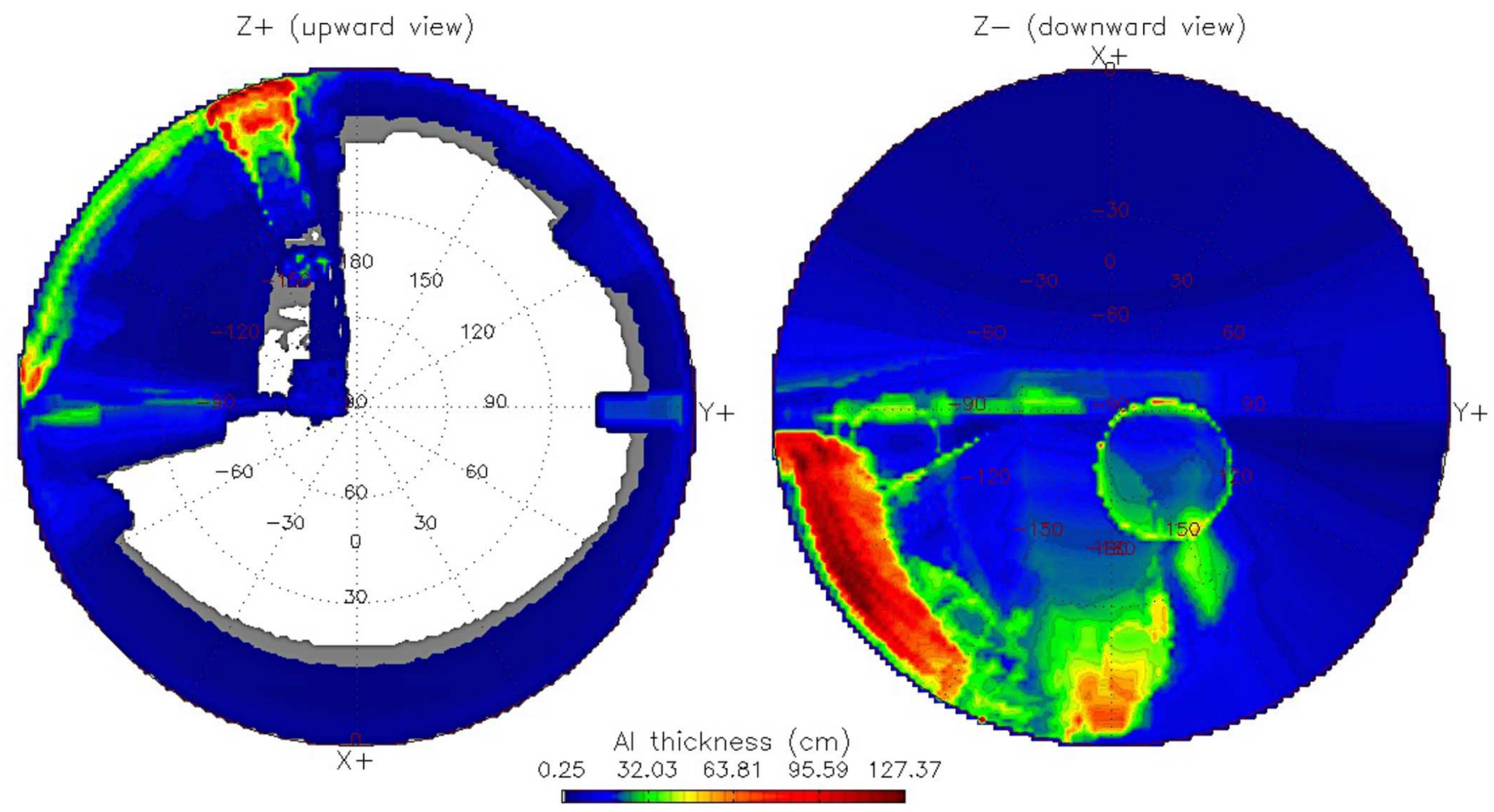

Fig. 6. Map representing the Al equivalent thicknesses around the RadFET located on the card MCE 1 (the less shielded one by surrounding materials). This map was computed with an angular resolution of $1^{\circ} \times 1^{\circ}$ and is displayed as a stereographic projection. The left panel corresponds to the upward view from the position of the RadFET, whereas the right panel corresponds to the downward view. The white region corresponds to the minimal Al equivalent thickness offered by the top cover of the electronic box.

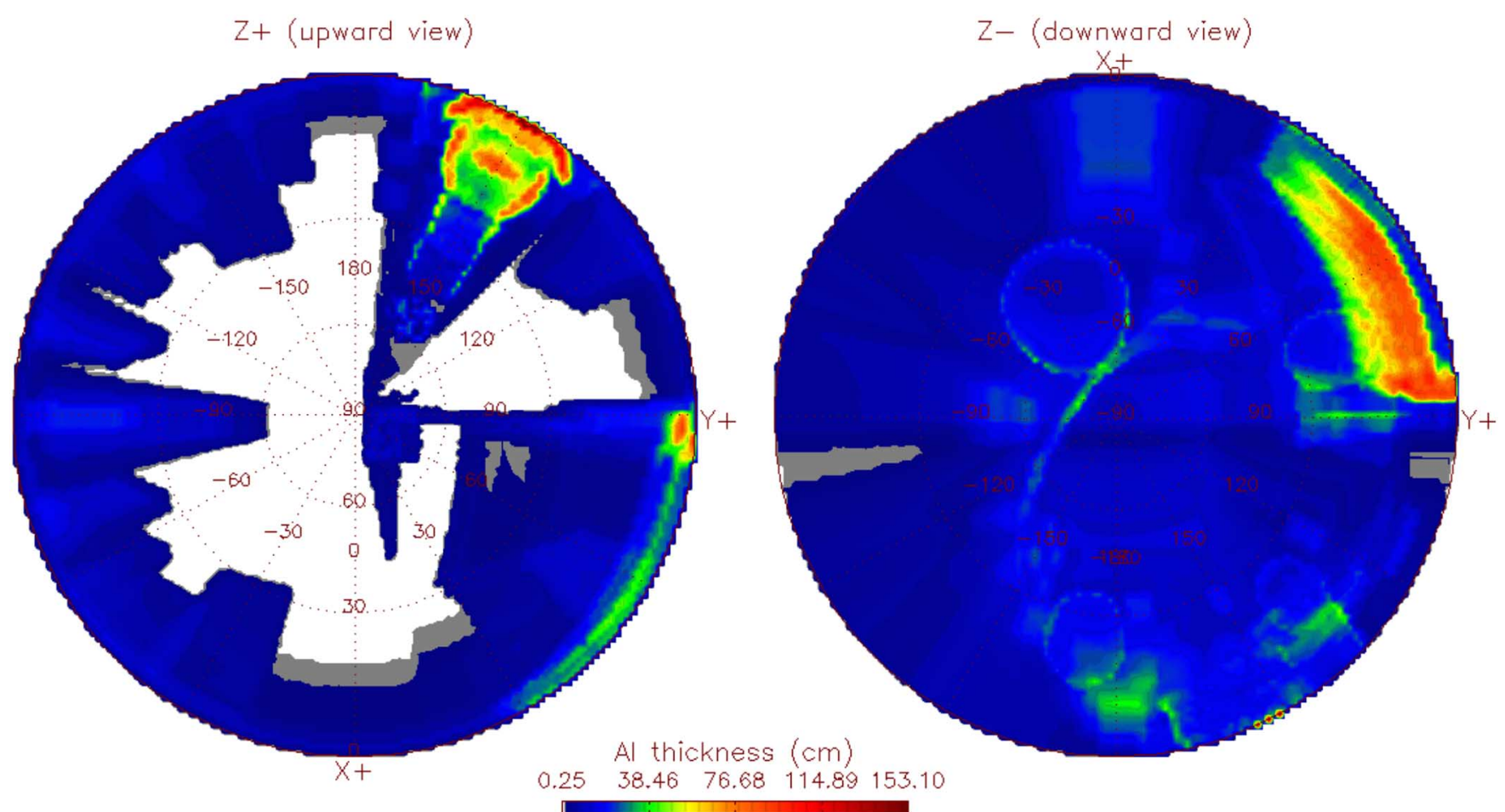

Fig. 7. Same as above for the RadFET located on the card MCE 6 (the most shielded one by surrounding materials).

\section{B. Dose Profile}

The dose profile curve was determined thanks to the Omere software [7] with the following environmental parameters:
AE8-MAX [8] and AP8-MIN [9] models for trapped particles corresponding to the worst case, plus ESP [10] model for solar particles with several confidence levels ranging in $80-95 \%$. In this study, we take care of the orbit evolution, and consider a non-constant dose profile curve all along the 12 years. Indeed, 


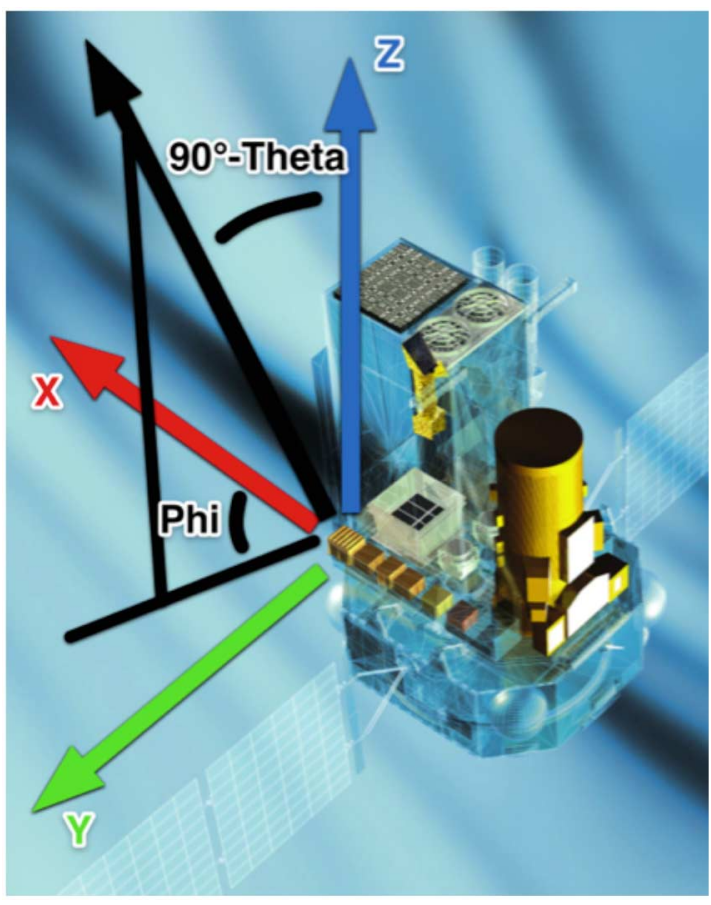

Fig. 8. Angular referential used in the sectorial analysis. The $\mathrm{X}$ and Z-axis point respectively $\pm 40^{\circ}$ towards the Sun and the astronomical target.

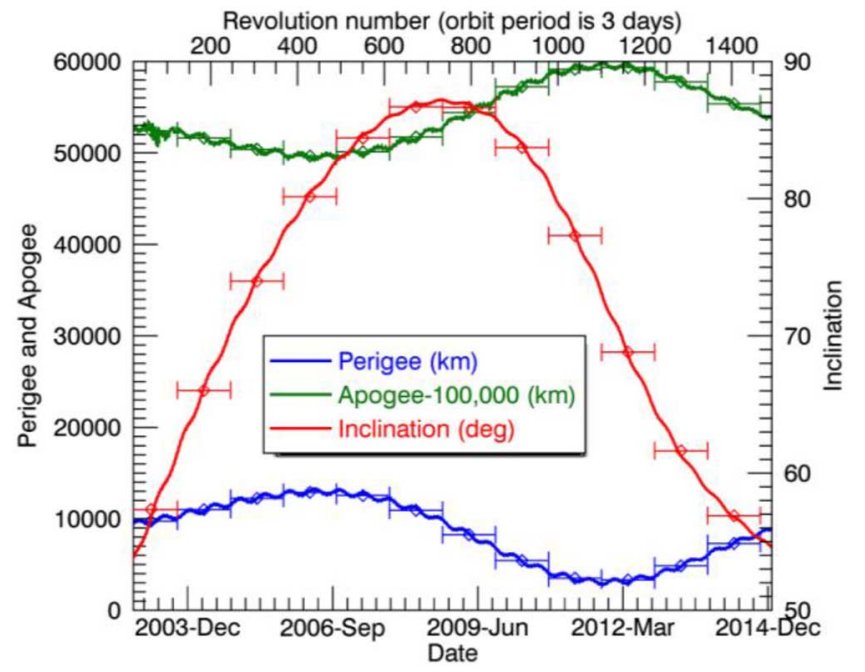

Fig. 9. Evolution of orbital parameters deduced from the actual flight orbit. An offset of $100,000 \mathrm{~km}$ has been subtracted to the apogee values in order to plot it on the same axis as the perigee.

during the first years of the mission, the trajectory crossed only the outer electron belt and just barely touched the inner belt with increased fluxes of protons. When the perigee became lower after a few years, increased fluxes of protons also contributed to the dose, as well as to displacement damages on solar cells, which has been observed through a decrease of the efficiency of the solar panels. So, in order to take into account the orbit evolution with the time, the orbit parameters were averaged for 1-year periods from the actual orbit (see Fig. 9). On this figure, we see that for 3 years between May 2010 and April 2013 , the perigee reached altitudes below $5,000 \mathrm{~km}$, which

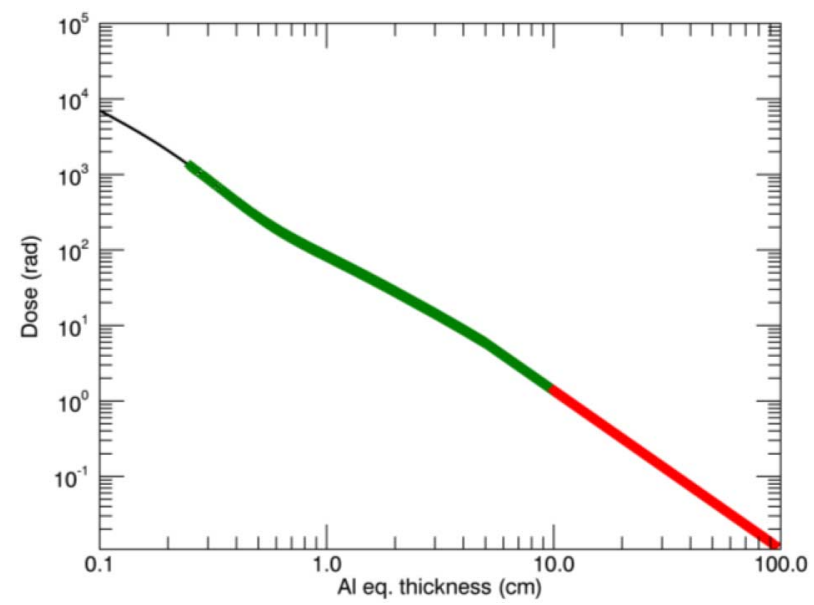

Fig. 10. Example of dose profile (black solid line) determined by SHIELDOSE2 with the geometry 'finite slab', plotted for the RadFET of the card MCE 1. Over plotted in green are the values of $\mathrm{Al}$ thicknesses that are in the range provided by the dose profile curve $(0.001-10 \mathrm{~cm})$. Other points plotted in red correspond to extrapolated values.

led the satellite to pass deeper inside the inner proton belt. In summary, for each set of environmental parameters (trapped particles provided by AP8MIN and AE8MAX models, solar particles provided by ESP model for 4 different values of the confidence level), 12 dose profiles were computed with Omere, one for each 1-year period after the launch (October 17, 2002). As an example, Fig. 10 shows the dose profile, calculated for a 1 -year period for the RadFET of the card MCE 1, which is the least protected by surrounding material.

Finally, 8 maps of Al equivalent thickness were computed with Geant3, one for each RadFET on-board INTEGRAL. The total dose through all elements of the $\mathrm{Al}$ equivalent thickness map determined above can be written as follows:

$$
\text { Dose }=\frac{\sum_{\theta, \emptyset} \text { DoseProfile }(\text { Thickness }(\theta, \emptyset)) \times d \Omega_{\theta, \emptyset}}{4 \pi}
$$

It is preferable to use the so-called 'finite slab' geometry of the SHIELDOSE2 model in (1), or eventually the 'semi finite slab' geometry. Indeed, in our scheme the particles that contribute to the dose are supposed to come from a limited solid angle $\left(1^{\circ} \times\right.$ $1^{\circ}$ ), corresponding to the angular resolution of our Al maps. A given dose profile is then used to convert in the same way each bin of these maps into dose maps, according to our hypothesis of omnidirectional average fluxes of particles introduced in the previous section. As a final result, our predicted dose evolution along 12 years of operations is displayed in Fig. 11.

The ESP model provides long-term estimated average fluences for solar max years and assumes no fluence for solar min years. This explains the zero ESP contribution during the period 2006-2011 (solar min) visible as the flat contribution in Fig. 11, whereas some solar proton events occurred during this period, as observed by IREM (see Fig. 4). At the right part of the figure, the final value of the prediction of the integrated dose reached after 12 years strongly depends on the confidence level considered for the solar contribution (ESP model). This is discussed in the following section. 


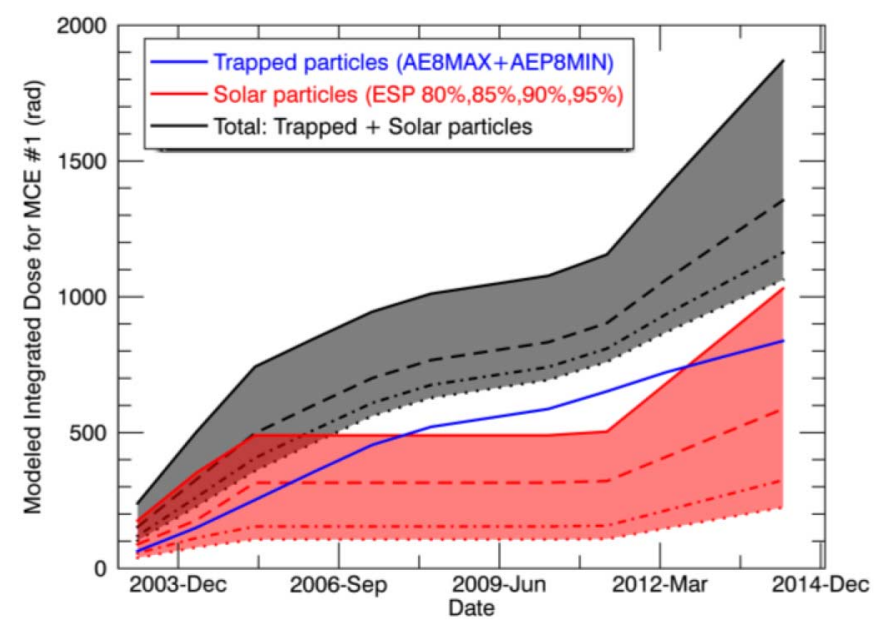

Fig. 11. Predicted cumulated dose (black) along 12 years of operations, superimposed with the contribution of only trapped particles (blue) and of solar particles (red).

TABLE I

\begin{tabular}{|c|c|c|c|}
\hline \multicolumn{4}{|c|}{ Al equivalent thickness (cm) } \\
\hline & $\min$ & mean & $\max$ \\
\hline MCE \#1 & 0.25 & 8.11062 & 127.366 \\
\hline MCE \#2 & 0.25 & 8.73918 & 161.239 \\
\hline MCE \#3 & 0.25 & 9.03564 & 134.312 \\
\hline MCE \#4 & 0.25 & 9.03418 & 116.757 \\
\hline MCE \#5 & 0.25 & 9.43219 & 174.581 \\
\hline MCE \#6 & 0.25 & 9.76972 & 153.100 \\
\hline MCE \#7 & 0.25 & 9.74138 & 151.413 \\
\hline MCE \#8 & 0.25 & 9.37137 & 151.526 \\
\hline \multicolumn{4}{|c|}{ Dose (rad) over 12 years } \\
\hline & & MCE 1-4 $(\mathrm{Y}+)$ & MCE 5-8 (Y-) \\
\hline $\mathrm{AE} 8 \mathrm{MAX}+\mathrm{A}$ & ESP $80 \%$ & 992 & 724 \\
\hline $\mathrm{AE} 8 \mathrm{MAX}+\mathrm{A}$ & ESP $85 \%$ & 1086 & 793 \\
\hline $\mathrm{AE} 8 \mathrm{MAX}+\mathrm{A}$ & ESP 90\% & 1266 & 926 \\
\hline $\mathrm{AE} 8 \mathrm{MAX}+\mathrm{A}$ & ESP $95 \%$ & 1745 & 1282 \\
\hline Mea & & 1150 & 890 \\
\hline
\end{tabular}

\section{RESUltS}

Our results are summarized in Table I, which displays the cumulative dose after 12 years in space since October 17, 2002. The first part of Table I gives the minimum, maximum and mean values of the $\mathrm{Al}$ equivalent thickness around each RadFET, as determined from our sectorial analysis. Note that the box MCE 1-4 (along the $\mathrm{Y}+$ axis) is less shielded than the box MCE 5-8 (along the $\mathrm{Y}-$ axis), since it is located more or less at a corner of the satellite platform unlike the other one, as visible in Fig. 5.

The second part of Table I displays the comparison of our calculated values (from sectorial analysis and environmental models) to the in-flight measured ones (RadFETs data). It is not so easy to make a direct comparison since it is strongly dependent on the confidence level considered for the contribution of solar particles. However, in-flight measurements show that the box containing RadFETs $1-4$ received a dose $30 \%$ higher than the other box containing RadFETs 5-8 due to less shielding materials around it. It is satisfying to see that our calculations lead exactly to the same ratio.

From Table I, it appears that a confidence level in the range $85-90 \%$ for the ESP model is adequate to estimate the dose reached on October 2014, after 12 years in space. But it is probably more pertinent to determine the best confidence level by considering the comparison for the whole mission, as displayed in Fig. 12, rather than only after 12 years of operation, as for Table I. From the general look of the curves, it appears that confidence level slightly higher (90-95\%) for the ESP model is more adequate. Because ESP is an average model and solar particle events are stochastic, it is not surprising that there is a mismatch the first couple of years, when not many solar events already occurred. From 2004 to 2011, the margin between the predicted dose and the measured one is progressively reduced as more and more solar events occur, and the model looks more consistent with the measurements. For the final years, the solar proton event actual contribution is probably less than that predicted by the ESP model likely because the current solar max is less active than the 3 solar cycles used to build ESP. The predicted integrated dose seems overestimating the measurement for this period, especially for $95 \%$ confidence level. However, the latter is more consistent with the recent dose increase observed in 2015 (but not shown here) partly due to recent solar activity. Unfortunately, because there is no possible confidence interval on the AE8/AP8 results used, our measurements do not allow to know which most contributes to the measurement-model difference: error in ESP's prediction of solar proton event dose, or error in AE8/AP8's prediction of trapped radiation dose. Resolving this is outside the scope of this paper, but a possible future work could consist in repeating the analysis with different models (e.g. AE9/AP9) or with dose derived from particle measurements (e.g. from IREM or GOES). In order to assess the error on our predicted dose values, we can consider an arbitrary offset of Al thickness, positive or negative constant to be added to our original Al maps, and then reprocess the whole chain of calculation. Of course, this process is not linear at all, but it is still worth mentioning that for small extra values of $\mathrm{Al}$ thickness, the error is about $100 \mathrm{rad}$ per $0.1 \mathrm{~mm}$.

\section{CONCLUSION}

The geometrical information available thanks to the measurements performed on the qualification model of the MCE box of INTEGRAL was revisited in order to determine the map of shielding materials around the RadFETs, which are still measuring the dose continuously after more than 12 years. This work allowed us to test the accuracy of the classical method to assess the expected dose, which consists of using sectorial analysis in combination with a dose profile curve issued from widely used environmental models. The comparison is good using standard parameters of classic environment models. The reader is referred to [11] and [12] for more details about radiation design margin. Our calculation of predicted radiation dose using (1) detailed modeling of the shielding geometry, (2) the actual orbit parameters as they evolve with time and (3) using the ESP solar particle flux at an appropriate confidence level and the classic AE8MAX/AP8MIN for trapped particles, leads to an accurate 


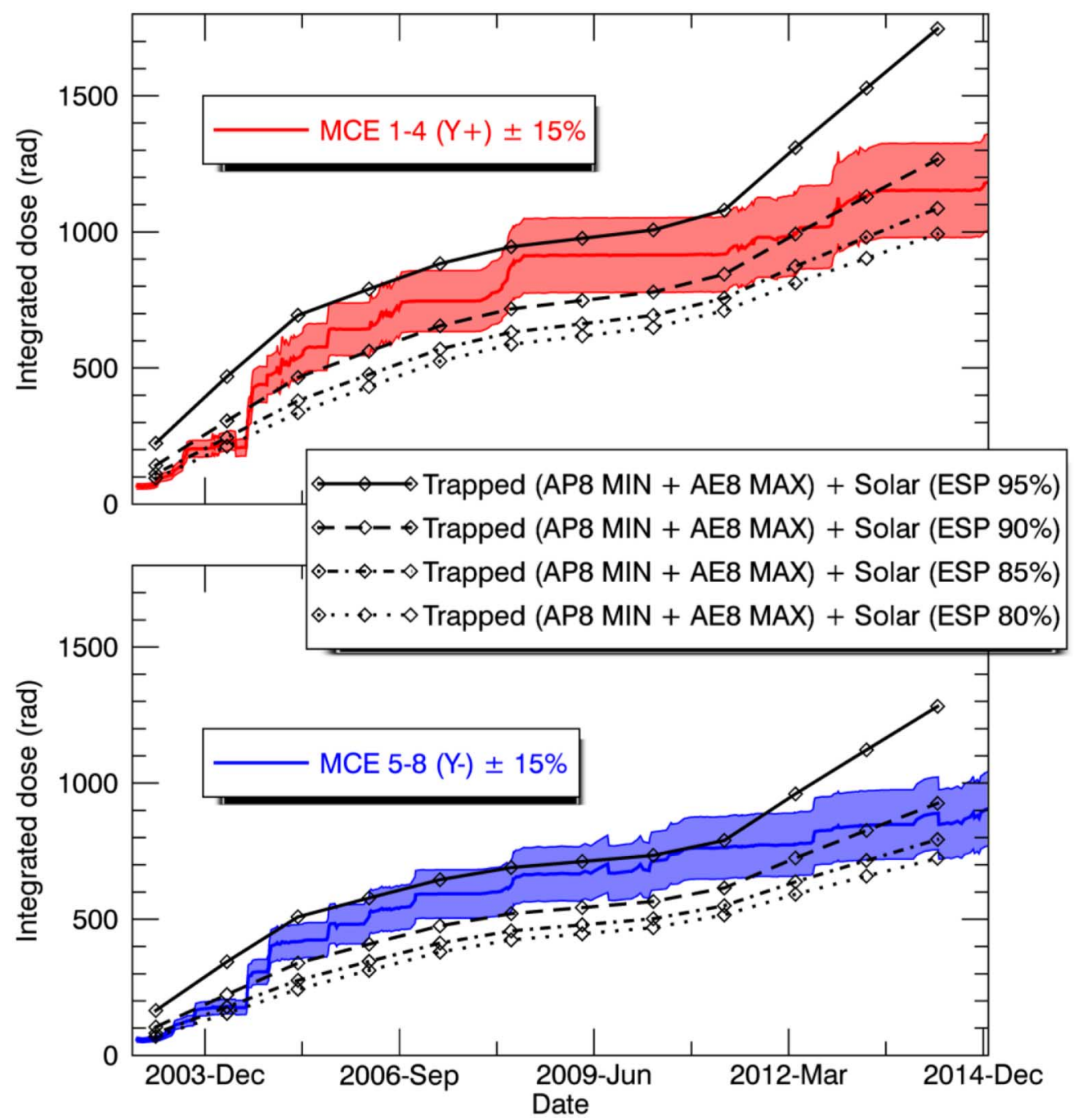

Fig. 12. Dose measurements averaged over 4 RadFETs located in each MCE box represented in red and blue color, compared with the predicted values of the integrated dose including trapped particles (AE8MAX+AP8MIN) plus solar particles (ESP with various confidence levels) represented as black lines. Dose measurements are represented with an arbitrary $15 \%$ relative error $(8 \%$ for the temperature variation + an arbitrary value of $7 \%$ for extra errors, as discussed in Section II).

value. Such an accurate prediction may be useful in particular when the problematic of the dose is stringent, and may allow reducing the design margin.

\section{ACKNOWLEDGMENT}

The authors thank François Daly (CEA) for providing us the qualification model of the MCE box, which allowed us to measure precisely the geometry and the implementation of RadFET, as well as for useful information on the RadFETs on-board INTEGRAL.

\section{REFERENCES}

[1] P. Ubertini, F. Lebrun, G. Di Cocco, A. Bazzano, A. J. Bird, K. Broenstad, A. Goldwurm, G. La Rosa, C. Labanti, P. Laurent, I. F. Mirabel, E. M. Quadrini, B. Ramsey, V. Reglero, L. Sabau, B. Sacco, R. Staubert, L. Vigroux, M. C. Weisskopf, and A. A. Zdziarski, "IBIS: The imager on-board INTEGRAL," Astron. Astrophys., vol. 411, pp. L131-L139, 2003.
[2] F. Lebrun, J.-P. Leray, P. Lavocat, J. Crétolle, M. Arquès, C. Blondel, C. Bonnin, A. Bouère, C. Cara, T. Chaleil, F. Daly, F. Desages, H. Dzitko, B. Horeau, P. Laurent, O. Limousin, F. Mathy, V. Mauguen, F. Meignier, F. Molinié, E. Poindron, M. Rouger, A. Sauvageon, and T. Tourette, "ISGRI: The INTEGRAL soft gamma-ray imager," Astron. Astrophys., vol. 411, pp. L141-L148, 2003.

[3] C. Labanti, G. Di Cocco, G. Ferro, F. Gianotti, A. Mauri, E. Rossi, J. B. Stephen, A. Traci, and M. Trifoglio, "The IBIS-PICSIT detector on-board INTEGRAL," Astron. Astrophys., vol. 411, pp. L149-L152, 2003.

[4] P. Gonçalves, A. Keating, A. Trindade, P. Rodrigues, M. Ferreira, P. Assis, M. Muschitiello, B. Nickson, and C. Poivey, "Modeling the response of the ESAPMOS4 RADFETs for the ALPHASAT CTTB experiment," IEEE Trans. Nucl. Sci., vol. 61, no. 3, pp. 1439-1443, Jun. 2014.

[5] W. Hajdas, P. Bühler, C. Eggel, P. Favre, A. Mchedlishvili, and A. Zehnder, "Radiation environment along the INTEGRAL orbit measured with the IREM monitor," Astron. Astrophys., vol. 411, pp. L43-L47, 2003.

[6] P. Laurent, O. Limousin, M. Cadolle-Bel, P. Goldoni, G. Malaguti, E. Caroli, G. De Cesare, M. Del Santo, A. J. Bird, J. Grygorczuk, and J. M. Torrejon, "Status of the Integral/IBIS telescope modeling and of the response matrices generation," Astron. Astrophys., vol. 411, pp. L185-L188, 2003.

[7] TRAD [Online]. Available: http://www.trad.fr/OMERE-14.html

[8] J. I. Vette, The AE-8 Trapped Electron Environment, Nov. 1991, NSSDC/WDC-A-RS 91-24, NASA-GSFC. 
[9] D. M. Sawyer and J. I. Vette, AP-8 Trapped Proton Environment for Solar Maximum and Solar Minimum, Dec. 1976, NSSDC/WDC-A-RS 76-06, NASA-GSFC.

[10] M. A. Xapsos, J. L. Barth, E. G. Stassinopoulos, E. A. Burke, and G. B. Gee, "Space environment effects: Model for emission of solar protons (ESP) - cumulative and worst case event fluences," NASA-Marshall Space Flight Center SEE Program, Dec. 1999 [Online]. Available: http://see.msfc.nasa.gov/publications/tp-1999-209763.pdf
[11] H. Garrett and I. Jun, "Spacecraft environment interactions," in Proc. NSREC Short Course, Las Vegas, NV, USA, Jul. 2011.

[12] R. Ecoffet, "On-orbit anomalies: Investigations and root cause determination," in Proc. NSREC Short Course, Las Vegas, NV, USA, Jul. 2011. 